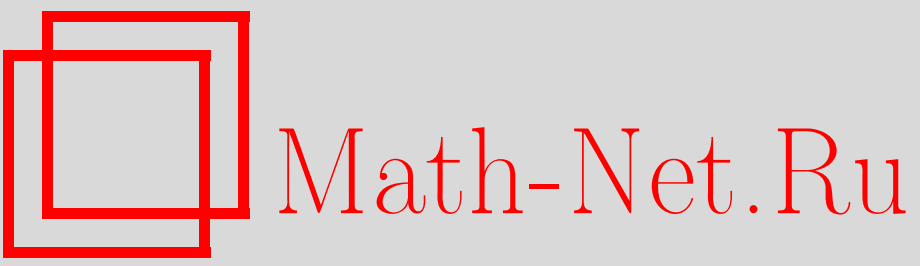

Т. К. Плышевская, Существование и единственность решений краевых задач для уравнений нейтрального типа, УМH, 1996, том 51, выпуск 6, 217-218

DOI: https://doi.org/10.4213/rm1035

Использование Общероссийского математического портала Math-Net.Ru подразумевает, что вы прочитали и согласны с пользовательским соглашением

http://www . mathnet.ru/rus/agreement

Параметры загрузки:

IP: 54.224 .187 .69

26 апреля 2023 г., 14:29:09 


\title{
СУЩЕСТВОВАНИЕ И ЕДИНСТВЕННОСТЬ РЕШЕНИЙ КРАЕВЫХ ЗАДАЧ ДЛЯ УРАВНЕНИЙ НЕЙТРАЛЬНОГО ТИПА
}

\author{
Т. К. ПлЫШЕВСКАЯ
}

Рассмотрим уравнение нейтрального типа

$$
\dot{x}(t)=f(t, x(h(t)), \dot{x}(\tau(t))), t \in[a, b], \quad x(\xi)=\dot{x}(\xi)=0, \xi \notin[a, b],
$$

где $f:[a, b] \times \mathbb{R}^{n} \times \mathbb{R}^{n} \rightarrow \mathbb{R}^{n}, h:[a, b] \rightarrow \mathbb{R}, \tau:[a, b] \rightarrow \mathbb{R}$.

Решением уравнения (1) будем называть абсолютно непрерывную (относительно меры Лебега $m) n$-мерную вектор-функцию, удовлетворяющую уравнению (1) почти всюду на $[a, b]$.

Пусть $T$ - локально компактное пространство в $\mathbb{R},\|\cdot\|$ - норма в $\mathbb{R}^{n}$. Через $L_{p}^{n}(T, \lambda)$ будем обозначать банахово пространство суммируемых в степени $p$ функций $x$ с нормой $\|x\|_{L_{p}^{n}(T, \lambda)}=\left(\int_{T}\|x(t)\|^{p} d \lambda(t)\right)^{1 / p}$, через $D_{p}^{n}([a, b])$ - банахово пространство абсолютно непрерьвных на $[a, b]$ вектор-функций $x$ таких, что $\dot{x} \in L_{p}^{n}([a, b], m),\|x\|_{D_{p}^{n}([a, b])}=$ $\max _{t \in[a, b]}\|x(t)\|+\|\dot{x}\|_{L_{p}^{n}([a, b], m)}$. Зададим краевые условия равенством

$$
l x \equiv \psi x(a)+\int_{a}^{b} \varphi(s) \dot{x}(s) d s=\gamma .
$$

Здесь $\psi$ - постоянная $(n \times n)$-матрица, $\operatorname{det} \psi \neq 0$, элементы $(n \times n)$-матрицы $\varphi$ принадлежат пространству $L_{q}^{1}([a, b], m)(1 / p+1 / q=1), \gamma \in \mathbb{R}^{n}$.

Тогда [1] краевая задача $\dot{x}(t)=y(t), l x=\gamma$ имеет единственное решение, определяемое равенством

$$
x(t)=\psi^{-1} \gamma+\int_{a}^{b}\left(C(t, s)-\psi^{-1} \varphi(s)\right) y(s) d s
$$

где $C(t, s)=\left\{\begin{array}{ll}0, & a \leqslant s \leqslant t \leqslant b, \\ 1, & a \leqslant t<s \leqslant b,\end{array}\right.$ а краевая задача $(1),(2)$ сводится к уравнению

$$
y(t)=f(t, \alpha+(H y)(t),(S y)(t)),
$$

где

$$
\begin{gathered}
(S y)(t)=\left\{\begin{array}{ll}
y(\tau(t)), & \tau(t) \in[a, b], \\
0, & \tau(t) \notin[a, b],
\end{array} \quad(H y)(t)=\int_{a}^{b} K(t, s) y(s) d s,\right. \\
K(t, s)=C(h(t), s)-\psi^{-1} \varphi(s), \quad \alpha=\psi^{-1} \gamma .
\end{gathered}
$$

Решение задачи $(1),(2)$ и уравнения (4) связаны равенством (3).

Обозначим $E=\{t \in T \subset \mathbb{R}: \tau(t) \in T\}$. Через $z_{E}$ обозначим сужение функции (или меры) $z$ на множество $E$, через $\tau(\lambda)$ - образ меры $\lambda$ при отображении $\tau$ [3].

Лемма. Пусть существуют положительная мера $\nu$ на $T \subset \mathbb{R}$ и число $p \in[1, \infty)$ maкuе, чmo:

1) функция $f(\cdot, u, v): T \rightarrow \mathbb{R}^{n} \quad \nu$-измерима при $u, v \in \mathbb{R}^{n},\|f(\cdot, \alpha, 0)\|^{p}$ - $\nu$-интегрируема нат и при всех $u_{1}, u_{2}, v_{1}, v_{2} \in \mathbb{R}^{n}$ и $\nu$-почти всюду на Т вьполняется неравенство $\left\|f\left(t, u_{1}, v_{1}\right)-f\left(t, u_{2}, v_{2}\right)\right\| \leqslant N\left\|u_{1}-u_{2}\right\|+M(t)\left\|v_{1}-v_{2}\right\|$, где $N \in \mathbb{R}$, $M: T \rightarrow \mathbb{R} \quad \nu$-измерима, $M(t) \geqslant 0$

2) пара $\left(\tau_{E}, M_{E}^{p}\right) \nu_{E}$-приспособлена [3] и существует $K>0$ mакое, что $\tau_{E}\left(M_{E}^{p} \nu_{E}\right)$ $\leqslant K \nu$

3) оператор $H: L_{p}^{n}(T, \nu) \rightarrow L_{p}^{n}(T, \nu)$ непрерьвен.

Тогда, если $N\|H\|_{L_{p}^{n}(T, \nu) \rightarrow L_{p}^{n}(T, \nu)}+K^{1 / p}<1$, то существует единственное в пространстве $L_{p}^{n}(T, \nu)$ решение уравнения (4).

Доказательство сводится к проверке условий принципа Банаха.

Приведем конструкцию меры $\nu$, которая обеспечивает выполнение условия 2) леммы. Подробно этот вопрос изложен в [4]. 
Теорема 1. Пусть $p \in[1, \infty), \lambda$ - положстельная мера на $T, M: T \rightarrow \mathbb{R}$ - ограниченная неотрицательная $\lambda$-измеримая функщия, пара $\left(\tau_{E}, M_{E}\right) \lambda_{E}$-приспособлена [3]. Пусть существуют числа $k \geqslant 0, \Delta \geqslant 0$ такие, что для любого $\lambda$-измеримого $A \subset T$ множество $\tau^{-1}(A) \cap\{t \in E: M(t)>0\} \quad \lambda$-измеримо и

$$
\lambda\left(\tau^{-1}(A) \cap\{t \in E: M(t)>0\}\right) \leqslant k \lambda(A)+\Delta .
$$

Тогда существует положительная мера $\nu$ на $T$ и число $K$ такие, что пара $\left(\tau_{E}, M_{E}^{p}\right)$ $\nu_{E}$-приспособлена $и \tau_{E}\left(M_{E}^{p} \nu_{E}\right) \leqslant K \nu$.

Доказано, что меру $\nu$ можно задать сходящимся рядом

$$
\nu=\sum_{n=0}^{\infty} \frac{\lambda_{n}}{\beta^{n}}, \quad \text { где } \beta>\max \{1, k\}, \quad \lambda_{0}=\lambda, \quad \lambda_{n+1}=\tau_{E}\left(M_{E}^{p} \lambda_{n_{E}}\right), \quad n=0,1, \ldots
$$

Структура меры $\nu$ зависит от структуры меры $\lambda_{1}=\tau_{E}\left(M_{E}^{p} \lambda_{E}\right):$ мера $\nu$ абсолютно непрерьвна относительно $\lambda$ тогда и только тогда, когда $\lambda_{1}$ абсолютно непрерывна относительно $\lambda$; мера $\nu$ имеет атомическую составляющую, независимую с $\lambda$, тогда и только тогда, когда $\lambda_{1}$ имеет аналогичную составляющую.

ТЕОРема 2. Пусть выполнены условия:

1) функция $f(\cdot, u, v):[a, b] \rightarrow \mathbb{R}^{n}$ т-измерима при $u, v \in \mathbb{R}^{n}$ и т-почти всюду на $[a, b]$ и всех $u_{1}, v_{1}, u_{2}, v_{2} \in \mathbb{R}^{n}$ вьполняется неравенство

$$
\left\|f\left(t, u_{1}, v_{1}\right)-f\left(t, u_{2}, v_{2}\right)\right\| \leqslant N\left\|u_{1}-u_{2}\right\|+M(t)\left\|v_{1}-v_{2}\right\|,
$$

где $M(\cdot)$ т-измерима и $0 \leqslant M(t) \leqslant K, N \in \mathbb{R}$;

2) функция $\tau_{E} m_{E}$-измерима и для любого $m_{E}$-измеримого е $\subset E$ множество $\tau^{-1}(e) \cap\left\{t \in E: M_{E}(t)>0\right\}$ m-измеримо.

Тогда: 1) для любого $p \in[1, \infty)$ и любого $K_{*}>K$ существует на $[a, b]$ положительная мера $\nu$ такал, что $\left.\tau_{E}\left(M_{E}^{p} \nu_{E}\right) \leqslant K_{*} \nu ; 2\right)$ если при этом мера $\lambda_{1}=\tau_{E}\left(M_{E}^{p} m_{E}\right)$ абсолютно непрерывна относительно $m$ или, кроме абсолютно непрерывной составляющей, содержит анатомическую меру,

$$
\int_{a}^{b}\|f(t, \alpha, 0)\|^{p} d \nu(t)<\infty, \quad I=\left(\int_{a}^{b}\left(\int_{a}^{b}\|K(t, s)\|^{p /(p-1)} d s\right)^{p-1} d \nu(t)\right)^{1 / p}<\infty
$$

$\left(\right.$ при $\left.p=1 \quad I=\int_{a}^{b} \operatorname{vrai}_{s \in[a, b]}\|K(t, s)\| d \nu(t)\right)$ u $N I+K_{*}^{1 / p}<1$, то на $[a, b]$ существует единственное в пространстве $L_{p}^{n}([a, b], \nu)$ решение уравнения (4).

\section{СПИСОК ЛИТЕРАТУРЫ}

[1] Азбелев Н. В., Максимов В. П., Рахматуллина Л. Ф. Введение в теорию функционально-дифференциальных уравнений. М.: Наука, 1991. [2] Драхлин М. Е., Плышевская Т. К. // Дифференц. уравнения. 1978. Т. 14. № 8. С. 1347-1361. [3] Бурбаки Н. Интегрирование. Меры, интегрирование мер. М.: Наука, 1967. [4] Плышевская Т. К. О разрешшмости функционально-дифференциальных уравнений в лебеговых пространствах. Магнитогорск: Магнитогорск. горно-металлург. ин-т, 1988. Деп. в ВИНИТИ 22.02.89, № 1186-В89.

Магнитогорский государственный педагогический институт
Принято редколлегией 18.04.1996 